Gdańsk 2018, Nr. 38

\author{
Andreas Klein \\ Würzburg, Deutschland \\ https://doi.org/10.26881/sgg.2018.38.06
}

\title{
Schreiben auf der Schwelle: Zum besonderen Charakter literarischer Beschreibungen des Unsagbaren in der Moderne
}

\begin{abstract}
Der vorliegende Beitrag richtet den Fokus auf die Schwierigkeiten moderner Schriftsteller, transzendente Erfahrungen konkret literarisch zu beschreiben. Im Schwellenzustand der Moderne verbleibt die Kunst als letzter Ort der Darstellung des Absoluten und transzendenter Phänomene. Angesichts des Vertrauensverlusts in religiöse Traditionen sind die Autoren in der Moderne gezwungen, in individueller Weise Worte, Symbole und Metaphern zu finden, um ihre Erfahrungen zur Sprache zu bringen und das Unsagbare doch zu sagen.
\end{abstract}

Schlüsselwörter: Literatur der Moderne, mystische Erfahrungen, Transzendenz

Writing on the Threshold: the Particular Character of Literary Descriptions of the Unsayable in Modernity. The present article focuses on the difficulties of modern writers to represent transcendental experiences in a tangible way. Given the liminal character of modernity, art and literature remain the last domains to display transcendent phenomena and the absolute in general. In face of loss of confidence in religious traditions modern authors are forced to find words, symbols and metaphors in their own individual way to describe their experiences of transcendence and at least to try to say the unsayable.

Keywords: literature, modernity, mystic experiences

Die Konfrontation des Individuums mit der Schwierigkeit, die Erfahrungsqualität mystischer Erlebnisse in konkrete Sprache und Worte zu kleiden, ist kein modernes Phänomen. In den Schriften antiker und mittelalterlicher Autoren, wie etwa beispielsweise Augustinus oder Meister Eckhart ${ }^{1}$, lassen sich Zeugnisse des unermüdlichen Versuchs finden, dem Namenlosen Namen zu geben und das Unsagbare zu sagen. Bei den Versuchen der Darstellung mystischer Erfahrungen konnten diese Autoren aus dem großen Sprachfundus der religiösen Begrifflichkeiten und Metaphern ihrer Tradition, in diesem Falle des Christentums, schöpfen. Trotz dieser Einbindung finden sich in den Schriften der jeweiligen Autoren Zeugnisse der immensen Schwierigkeit, inkommensurable Erlebnisse der Transzendenz in Worte zu fassen und angemessen zu schildern.

Die Schwierigkeit, das Erlebte in Worte zu fassen, ist ein wesentliches Merkmal der mystischen Erfahrung. Dieses Merkmal ist eines der wenigen verbindenden Elemente der unterschiedlichen Definitionen von Mystik. Sonst gehen die Meinungen darüber, wie Mystik

1 Vgl. dazu Augustinus, Bekenntnisse, Berlin, Darmstadt 1968, S. 208-210. Ebenso Meister Eckhart, Predigt 26, in: ders., Werke I. Texte und Übersetzungen von Josef Quint. Herausgegeben und kommentiert von Niklas Largier, Frankfurt a.M. 2006, S. 295-303. 
oder die mystische Erfahrung definiert werden kann, weit auseinander. ${ }^{2}$ Im Kontext dieses Aufsatzes soll die mystische bzw. transzendente Erfahrung wie folgt gekennzeichnet werden. Ein mystisches bzw. transzendentes Erlebnis ist eine Erfahrung, die sich in ihrer Qualität radikal vom Alltagserleben des Individuums abhebt. Das Individuum erlebt dabei ein Übersteigen der eigenen Person und eine Öffnung zu einer, oder gar eine Vereinigung mit einer transpersonalen Entität (Gott, das Absolute, das Wirklichkeitsganze). Mit dieser Erfahrung können intensive affektive Zustände unterschiedlicher Art einhergehen. Das Ziel dieses Beitrages ist es, zuerst aufzuzeigen, dass in der Moderne nun Literaten zu legitimen Vermittlern transzendenter und mystischer Erfahrungen werden. Ebenso soll beschrieben werden, mit welchen spezifischen Hindernissen und Schwierigkeiten moderne Schriftsteller und Dichter bei ihren Versuchen, das Unsagbare zur Sprache zu bringen, konfrontiert waren. Im Zuge der nachfolgenden Darstellung soll in eklektischer Weise auf Denker und Theoretiker verschiedenster Provenienz zurückgegriffen werden, um die angesprochenen Aspekte und Betrachtungsweisen zu untermauern.

In einem langwierigen Prozess, der sich über mehrere Jahrhunderte erstreckt, werden die religiösen Metaphern und Begriffe immer fragwürdiger in ihrem Gehalt. Dabei wird die Sphäre des Religiösen als Ursprung und Ort des Unsagbaren kritisch hinterfragt und damit immer mehr aus dem Bewusstsein des Menschen herausgedrängt. Dieser Prozess steigert sich fortwährend bis er in der Moderne manifest und akut wird. Das rasante Voranschreiten auf dem Gebiet der Technik, die Errungenschaften der Naturwissenschaften, Darwins Evolutionstheorie und viele andere gesellschaftliche Entwicklungen dienen als Katalysatoren für diese progressive Dynamik der Unterminierung ehemals als fest und gesichert geglaubter religiöser Strukturen. Die von Georg Lukács in seiner Romantheorie diagnostizierte „transzendentale Obdachlosigkeit “3 als vorscheinendes Charakteristikum der modernen Welt kann als prägnante und treffende Bestimmung des Endresultats des beschriebenen Prozesses gelten. Lukács` Formulierung bildet das Eingangstor zu einem Verständnis für die Besonderheit von modernen, literarisierten Darstellungen von mystischen Erfahrungen. In der Rede von der transzendentalen Obdachlosigkeit verdichtet sich das religiöse und metaphysische Dilemma der Moderne. Die einstigen Formen, welche über Generationen tradiert, benutzt und gehandhabt wurden, um metaphysische bzw. religiöse Erfahrungen und Erlebnisse zu kanalisieren und zu vermitteln, sind zerbrochen und können ihren Dienst nicht mehr leisten. Friedrich Nietzsches Spruch vom Tode Gottes ${ }^{4}$ markiert dabei nur den ekstatischen Moment des drängenden Bewusstwerdens eines Prozesses, der sich manchmal verborgen, manchmal direkt offen ersichtlich, schon durch die Geistes- und Kulturgeschichte der vorhergehenden Jahrhunderte gezogen hat.

2 Zur Geschichte des Begriffs Mystik vgl. Josef Sudbrack, Mystik. Sinnsuche und die Erfahrung des Absoluten, Darmstadt 2002, S. 15-18.

3 Georg Lukács, Die Theorie des Romans. Ein geschichtsphilosophischer Versuch über die Formen der großen Epik, Darmstadt, Neuwied 1982, S. 47.

4 Friedrich Nietzsche, Die fröhliche Wissenschaft, in: ders., Morgenröte. Idyllen aus Messina. Fröhliche Wissenschaft. Kritische Studienausgabe, Bd. 3. Herausgegeben von Giorgio Colli und Mazzino Montinari, Berlin, New York, München 1988, S. 343-653, hier: S. 480-482. 
Es ist ebenfalls Nietzsche, der in seinem vielgestaltigen, teilweise prophetischen Denken den letztmöglichen Ort für eine Auseinandersetzung mit Ideen und Phänomenen der Transzendenz aufweist: nämlich die Kunst. Als die „metaphysische Tätigkeit“ ${ }^{\text {“5 }}$ des Lebens ist die Kunst das letzte Refugium in der Moderne, in dem die Begegnung mit transzendenten Kräften beschrieben und adäquat dargestellt werden kann. Prominente Vorläufer für Nietzsches Bestimmung der Aufgabe der Kunst sind die Dichter Friedrich Hölderlin und Arthur Rimbaud. In ihren Werken finden sich konkrete Überlegungen zum Auftrag des Dichters und seines Schaffens. So schreibt Hölderlin im Gedicht „Wie wenn am Feiertage...“, dass es den Dichtern allein gebührt „unter Gottes Gewittern [...] mit entblößtem Haupt zu stehen“6, um dem „Volk ins Lied / gehüllt die himmlische Gabe zu reichen. ${ }^{\text {"7 }}$ Rimbaud fordert in seinen Seher-Briefen, dass der Dichter sich selbst zum Seher verwandeln muss. Dies gelingt, indem er sich selbst erkennt und seine Seele erforscht und dann vervollkommnet. Der Dichter muss zum Unbekannten durchdringen und von den Visionen, die er dort erhält, künden. Rimbaud fordert die Dichter auf, den Sprung zu wagen, hinzu den „unerhörten und unsagbaren Dingen." ${ }^{\text {8 }}$ Man kann hier eine gegenläufige Bewegung beobachten: Verlieren also religiöse Metaphern und Begriffe in der anbrechenden Moderne zunehmend ihre Glaubwürdigkeit und sind somit nicht mehr tauglich als Träger einer Darstellung mystischer bzw. transzendenter Erfahrungen, welche die Schwelle des Sagbaren übersteigen, so gewinnen literarische Zeugnisse und künstlerische Darstellungen eben dieser Phänomene immer weiter an Bedeutung. Diese literarisierten Beschreibungen sind somit legitime und ebenbürtige Zugänge zu mystischen bzw. transzendenten Erfahrungen.

Auch der Philosoph Hans Blumenberg teilt diese Einschätzung der Rolle und Aufgabe der Kunst in der Moderne. Im modernen „Zeitalter, das sich in seinem alles durchdringenden Willen zu historischem Verstehen und Sich-verstehen seine Unbefangenheit genuinen Ausdrucks fast überall zerstört hat “9, bleibt die Kunst für Blumenberg ein „Reservat des historisch bewußten Ahistorismus " ${ }^{10}$. Auch ihm gilt die Kunst als verbleibender Darstellungsort transzendenter Erfahrungen, die sich auf eine das Alltagserleben übersteigende Wirklichkeit beziehen. „Das Absolute, das dem modernen Menschen am ehesten in seinen ästhetischen Erfahrungen zu begegnen scheint [...], präsentiert sich hier als das von der Gebrochenheit des

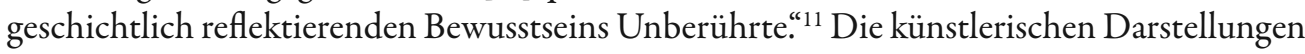

5 Friedrich Nietzsche, Vorwort an Richard Wagner. Die Geburt der Tragödie. Oder: Griechenthum und Pessimismus, in: ders., Die Geburt der Tragödie. Unzeitgemäße Betrachtungen I-III (1872-1874). Nietzsches Werke. Kritische Gesamtausgabe. Giorgio Colli und Mazzino Montinari. Abt. 3, Bd. 1, Berlin 1972, S. 5-150, hier: S. 20. Zu Nietzsches Kunstauffassung siehe weiter: Theo Meyer, Nietzsche und die Kunst, Tübingen 1993, S. $18-145$.

6 Friedrich Hölderlin, Wie wenn am Feiertage, in: ders., Sämtliche Gedichte, Herausgegeben von Detlev Lüders, 1989 Wiesbaden, S. 300-302, hier: S. 302.

7 Ebd.

8 Arthur Rimbaud, Rimbaud an Paul Demeny, in: ders., Sämtliche Dichtungen. Zweisprachige Ausgabe, 2004 München. S. 369-379, hier: S. 373.

9 Hans Blumenberg, Paradigmen zu einer Metaphorologie. Kommentar von Anselm Haverkamp, Frankfurt a.M. 1998, S. 28. Siehe die dortige Fußnote.

10 Ebd.

11 Ebd. 
transzendenter, mystischer Erfahrungen von Dichtern und Literaten sind auch für Blumenberg adäquate Platzhalter für die leere Stelle, die sich durch den Verlust der Überzeugungskraft tradierter und etablierter Gewissheiten und Grundsätze aufgetan hat.

Diesen Erkenntnissen folgend untersucht Blumenberg in frühen kleineren Arbeiten zur Literatur, wie sich das Verhältnis von transzendenter Erfahrung und dem Ringen nach einer Versprachlichung dieser Erfahrung in Anbetracht eines zerbrochenen oder nichtvorhandenen religiösen Bezugsrahmens konkret literarisch manifestiert. In seinem Aufsatz „Der absolute Vater“, in dem er sich mit Franz Kafkas „Brief an den Vater" auseinandersetzt, arbeitet Blumenberg noch einmal klar und eindeutig am Beispiel Kafkas das Dilemma der Moderne heraus. Blumenberg zufolge scheitert Kafka daran, sein „tief verwurzeltes Transzendenzbewußsein “12

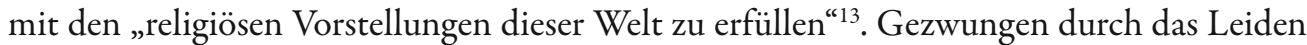
an der Namenlosigkeit und der "Leere dieser gottlosen Religiosität“14, versucht Kafka dieses Vakuum mit den „Bildern und Symbolen seiner Dichtungen“" ${ }^{15}$ zu besetzen. Gemäß Blumenberg geschieht Kafkas Schreiben nicht aus einem schöpferischen Überfluss heraus, sondern ist der genuine Ausdruck einer geistigen Existenzbehauptung. ${ }^{16}$ Kafka dichtet gegen die moderne transzendentale Obdachlosigkeit an. Er versucht, die drückende Leere mit seinen literarischen Bildern und Symbolen im wörtlichen Sinne zu verdichten. In seinen Texten spiegelt sich das „Schicksal einer Epoche, deren Bezug zum Absoluten sich an den überlieferten Gehalten nicht mehr erfüllen zu können scheint ${ }^{\text {"17 }}$.

Blumenbergs Beschreibung der Motivation von Kafkas Schreiben steht exemplarisch für die Situation vieler moderner Schriftsteller, die in ihren Texten angesichts einer gefühlten transzendenten Obdachlosigkeit damit ringen, Erfahrungen, welche die Alltagswirklichkeit übersteigen, ohne den fraglosen Rückgriff auf geläufige und tradierte Weisen, stimmig sprachlich darzustellen. Der moderne Schriftsteller ist dabei einer doppelten Sprachlosigkeit ausgesetzt. Zunächst ist es die fundamentale Erfahrung selbst, welche sich signifikant von den Phänomenen, mit denen das Alltagsbewusstsein umgehen muss, abhebt, die ihn sprachlos macht. Dazu kommt, dass er, um das Erlebte einordnen und beschreiben zu können, nicht fraglos auf das Reservoir an Wörtern, Begriffen und Metaphern aus den zweifelhaft gewordenen religiösen Zugängen zurückgreifen kann. Der moderne Schriftsteller ist somit auch immer auf der Suche nach markanten Wörtern, Wortfeldern oder Metaphern aus bestimmten Regionen der Kultur oder der Geisteswissenschaft, die ihm Material liefern, um die Erfahrung adäquat auszudrücken.

Paradoxerweise stammt dieses Material zum Teil dann doch wieder aus religiösen, vor allem christlichen Traditionen. Dabei werden die Begriffe und Metaphern in neuer Weise, welche sich vom ursprünglichen, traditionellen Gebrauch der Wörter abhebt, verwendet. Es ist, als arbeiteten sich manche Dichter und Schriftsteller am metaphysischen Echo dieser Begriffe und Metaphern ab. Trotz der modernen Zweifel besitzen sie noch genügend Ausstrahlungskraft,

12 Hans Blumenberg, Der absolute Vater, in: „Hochland“ 45 (1952/53), S. 282-284, hier: S. 283.

13 Ebd.

14 Ebd.

15 Ebd.

16 Vgl. ebd.

17 Ebd., S. 284 
um als Wegweiser auf den Bereich des Unsagbaren und Überweltlichen zu zeigen. Ein passendes Beispiel hierfür ist die Verwendung des Engels in Rainer Maria Rilkes „Duineser Elegien“ oder in seinem Gedicht „Der Engel“. Der Engel ist Rilkes Symbol für eine Wirklichkeit, die die kleine Alltagswirklichkeit, auf welche das menschliche Bewusstsein normalweiser geeicht ist, gewaltig übersteigt und überdauert. Durch des Engels „Herz geht riesig aufgerichtet / das ewig kommende, das kreist. ${ }^{18}{ }^{18}$ Der Engel erscheint bei Rilke als eine Mittlerfigur in eine transzendente Sphäre, ohne dass dabei diese Sphäre explizit mit einer bestimmten Gottesvorstellung assoziiert wird. Damit fungiert der Engel als Stellvertreter für das Unsagbare und Transzendente. In den Elegien ist der Engel mit seinen ambivalenten Wesenszügen des Schreckens und der Schönheit der transzendente Bezugspunkt, an dem das diesseitige menschliche Wirken und Streben gemessen werden kann. Angesichts der übermenschlichen Größe des Engels gewinnt gerade das „Einfache, das von Geschlecht zu Geschlechtern gestaltet ${ }^{\text {"19 }}$ seinen Wert und seine wahre Größe.

Der intertextuelle Rückbezug auf die religiöse Tradition hat viele Gesichter. Er kann wie bei Rilke in Form einer reflektierten Wiederaufnahme des religiösen Sprachmaterials geschehen, wobei dessen Konnotation verändert und variiert wird. Ebenso werden religiöse Begriffe oder Metaphern verwendet, ohne dass diese dabei ihren ehemals religiösen Sinn einlösen können. So ist zum Beispiel der Gott in den Dichtungen Georg Trakls kein Gott der Gnade und der Barmherzigkeit, sondern bleibt abwesend von dieser als kalt empfundenen Welt. ${ }^{20}$ Trakls Gott ist stumm, und „Gottes Schweigen“21 ist in vielen Gedichten Trakls präsent. Dabei benutzt Trakl immer wieder intertextuelle Bezüge zur Religion des Christentums. Er gibt den Gedichten religiöse Titel wie „Psalm“ oder „Geistliches Lied“. Diese Werke können aber die titelverheißenden Erwartungen nicht einlösen. Im Gegenteil, Trakl malt mit seiner bildhaften Sprache apokalyptische Szenarien des Verfalls und der Hoffnungslosigkeit. „Die toten Waisen liegen an der Gartenmauer ${ }^{\text {“22 } 2}$ und „in der Düsternis des alten Asyls verfallen menschliche Ruinen “23. Trakls Psalm spendet keinen Trost mehr und stärkt auch nicht den Glauben, er strahlt nur Hoffnungslosigkeit und düstere Verzweiflung aus. Es wird deutlich, dass Trakl und auch Rilke aus demselben Impuls heraus, wenn auch auf verschiedene Weise, schreiben. Sie ,gehören gleichermaßen zu den unzähligen Erben Nietzsches und teilen - wie das Gros ihrer Zeitgenossen - das Bewusstsein vom Tod Gottes. ${ }^{\text {“2 } 24}$ Damit partizipieren sie „am unaufhaltsamen Prozess der Säkularisierung, der paradoxerweise mit der Sakralisierung der Kunst einhergeht." ${ }^{25}$

Einen ganz anderen Weg des intertextuellen Rückbezugs wählt Robert Musil. Dieser setzt sich für seinen Roman „Der Mann ohne Eigenschaften“ intensiv mit den historischen

18 Rainer Maria Rilke, Der Engel, in: ders., Neue Gedichte, Leipzig 1907, S. 42.

9 Ders., Die neunte Elegie, in: Rainer Maria Rilke, Duineser Elegien, Leipzig 1923, S. 33-35, hier: S. 35.

Vgl. Johannes Klein, Kampf um Gott in der deutschen Dichtung, Witten, Berlin 1974, S. 390-392.

Georg Trakl: De profundis, in: ders., Gedichte, Leipzig 1913, S. 38.

Ders.: Psalm, in: ders., Gedichte, Leipzig 1913, S. 47-48, hier S. 48.

Ebd.

24 Gerald Stieg, Sakralisierung durch Dichtung bei Rainer Maria Rilke und Georg Trakl, in: Albert Meier, Alessandro Cosstazza, Gérard Laudin (Hrsg.), Kunstreligion. Ein ästhetisches Konzept der Moderne in seiner Entfaltung, Bd. 2: Die Radikalisierung des Konzepts nach 1850, Berlin, Boston 2012, S. 265-279, hier: S. 267. 25 Ebd. 
Zeugnissen mystischer Bekenntnisse auseinander und benutzt dazu Martin Bubers Sammlung „Ekstatische Konfessionen“. ${ }^{26}$ Die Beschäftigung mit den vielfältigen Formen von mystischen Bekenntnissen, die aus unterschiedlichen geschichtlichen Situationen stammen, wird an vielen Stellen in Musils Text ersichtlich. ${ }^{27}$ Die Suche des Protagonisten Ulrich nach Formen einer taghellen Mystik und dem anderen Zustand ist eines der grundlegenden Motive des Romans. Besonders deutliche Spuren von Musils Auseinandersetzung mit den klassischen Darstellungen mystischer Erlebnisse findet man in dem aus dem Nachlass stammenden und mehrfach überarbeiteten Kapitel mit dem Titel „Atemzüge eines Sommertages“. Dort finden sich klassische Elemente einer mystischen Erfahrung wieder. So zum Bespiel das profunde Erlebnis der Verschmelzung des Individuums mit etwas Höherem, der Vereinigung von Innen und Außen: Es ist, ,als wäre ein Keil ausgesprungen, der die Welt geteilt hat. ${ }^{\text {“28 }}$ Darüber hinaus beschreibt Musil die dabei empfundenen Gefühle von „Entzückung “ ${ }^{29}$ und „Verklärung “30. Auch der Aspekt der Zeit, das für das mystische Erlebnis charakterisierende Ineinandergreifen von Ewigkeit und Jetzt, wird von Musil dichterisch gestaltet: „Die Zeit stand still, ein Jahrtausend wog so leicht wie ein Öffnen und Schließen des Auges, sie war ans Tausendjährige Reich gelangt, Gott gar gab sich vielleicht zu fühlen. ${ }^{\text {“31 }}$ Musil hebt bei dieser Literarisierung eines mystischen Erlebnisses klar die positiv affektiven Begleiterscheinungen der Erfahrung hervor und (re-)kreiert damit das klassische Bild der unio mystica, welches die Einheitserfahrung mit einer nie gekannten, profunden Gefühlsstimmung der Glückseligkeit oder einer allumfassenden Liebe verbindet.

An den anderen genannten Beispielen literarischer Annäherungen an das Unsagbare wird ersichtlich, dass Musils Form der Beschreibung in dem Kapitel „Atemzüge eines Sommertages“ nur eine Nuance der verschiedenen Schattierungen darstellt, mit welchen ein transzendentales Erlebnis textlich und sprachlich ausgestaltet werden kann. Die transzendente Erfahrung besitzt einen ambivalenten Charakter. Tiefe Glückseligkeit, Einheitserfahrungen von Individuum und Gott bzw. Welt, oder das Gefühl einer alle Dinge durchströmenden Liebe und Verbundenheit gehören zur einen Seite der möglichen affektiven Beschaffenheit eines transzendenten Erlebnisses. Doch die Konfrontation mit dem Unsagbaren kann auch negative Gefühle und Emotionen auslösen. Der Engel Rilkes in den „Duineser Elegien“ besitzt auch eine Seite des Schreckens: „Ein jeder Engel ist schrecklich“. ${ }^{32}$ Er verfügt über eine gewaltige Präsenz, welche das nach dem Engel rufenden lyrische Ich demütig und ohnmächtig werden lässt. „Der Lockruf dunkelen Schluchzens ${ }^{\text {“33 }}$ wird zunächst verschluckt aus Angst vor dem

26 Vgl. Dietmar Goltschnigg, Mystische Tradition im Roman Robert Musils. Martin Bubers „Ekstatische Konfessionen“ im „Mann ohne Eigenschaften“, Heidelberg 1974, S. 63-64.

27 Wie Goltschnigg aufweist, verwendet Musil dazu verschiedenen Techniken des Zitierens, wie offenes oder verdecktes Zitat, Entlehnung und Modifizierung, sowie Variation und Kontamination. Vgl. dazu: Dietmar Goltschnigg, Mystische Tradition, S. 114-119.

28 Robert Musil, Der Mann ohne Eigenschaften. Band 2. Aus dem Nachlass. Herausgegeben von Adolf Frisé, Reinbek bei Hamburg 2009, S. 1234.

29 Ebd. S. 1233.

30 Ebd.

31 Ebd.

32 Rainer Maria Rilke, Die erste Elegie, in: ders., Duineser Elegien, Leipzig 1923, S. 7-10, hier S. 7.

33 Ebd. 
„stärkeren Dasein “" ${ }^{34}$ des Engels. Ein weiteres Beispiel ist der zuvor beschriebene deus absconditus aus Trakls Gedichten. Dieser lässt das lyrische Ich voller Hoffnungslosigkeit und Verzweiflung allein zurück in einer bedrohlichen und kalten Welt.

Im Anschluss an die einflussreichen religionswissenschaftlichen Arbeiten Rudolf Ottos ist der ambivalente Charakter mystischer Erfahrungen und transzendenter Erlebnisse zu berücksichtigen. Das Numinose als Kern der religiösen Erfahrungen kann sich in kontrastiven Momenten zeigen. ${ }^{35}$ Entscheidend ist dabei, zu verstehen, dass sich diese Momente immer in ambivalenter Weise über die Affekte und Gefühle des Individuums äußern. ${ }^{36}$ Neben positiven Aspekten wie Hingezogenheit, Faszination zu etwas Überweltlichem bzw. Göttlichem $^{37}$ stehen auch negative Seiten der Erfahrung, wie zum Bespiel ein allumfassendes Ohnmachtsgefühl ${ }^{38}$ oder gar Gefühle von Schrecken und Schauer ${ }^{39}$. Die Konfrontation mit einer die Alltagswirklichkeit übersteigenden, gänzlich anderen Realität kann schlussendlich ebenso eine fundamentale Angst auslösen. Otto gibt eine breite Palette von unterschiedlichen, teils gegensätzlichen Gefühlsregungen und Affekten an, welche während der Konfrontation mit dem Numinosen bzw. bei transzendenten Erfahrungen vom Individuum durchlebt werden können. Ottos Darlegungen sensibilisieren für die Tatsache, dass sich auch in modernen literarischen Beschreibungen von Transzendenzerfahrungen unterschiedliche, teilweise auch kontrastive Affekte aus einem breiten Spektrum der Gefühlsregungen finden lassen.

Diese Tatsache ist ein Faktor, der zum wesentlichen Kennzeichen der literarisierten Transzendenzerfahrungen in der Moderne beiträgt, nämlich ihrer Vielfältigkeit und Individualität. Jede dieser Beschreibungen ist in ihrer je spezifischen Weise einzigartig und einmalig. Sie unterscheiden sich nicht nur hinsichtlich der beschriebenen Gefühlsregungen, sondern auch in der mannigfaltigen Art des Zugriffs auf das in der Moderne fragwürdig gewordene Sprachmaterial aus den religiösen Traditionen. Hinzu kommen die großen Unterschiede in den konkreten Strategien der sprachlichen Realisierung und Darstellung der Erfahrungen. In jeder literarisierten Annäherung an den Bereich des Unsagbaren offenbart sich ein dem Dichter und auch ein der konkreten Person je eigener und partikulärer Weg. Die Texte der Schriftsteller zeigen in genuin einzigartiger Weise die verschiedenen Facetten transzendenter Erfahrungen auf. Ihre Individualität verlangt es, dass man sich jedem Text, jeder Beschreibung einzeln nähern und in genauer Untersuchung die jeweils betonten und aufgezeigten Facetten des Unsagbaren herausarbeiten muss.

Mit Rückgriff auf Victor Turners ursprünglich aus der Ethnologie stammendes Konzept der Liminalität lässt sich die Besonderheit der Situation moderner Schriftsteller bei der Darstellung des Unsagbaren sowie der Beschreibung transzendenter Erfahrungen weiter akzentuieren. Turner übernimmt dabei eine von Arnold van Gennep erarbeitete Dreigliederung

34 Ebd.

35 Vgl. Rudolf Otto, Das Heilige. Über das Irrationale in der Idee des Göttlichen und sein Verhältnis zum Rationalen, München 2004, S. 56-57.

36 Vgl. Carsten Colpe, Über das Heilige. Versuch, seine Verkennung kritisch vorzubeugen, Frankfurt a.M. 1990, S. 45 und S. 52

37 Vgl. Rudolf Otto, Das Heilige, S. 42.

38 Vgl. ebd. S. 9.

39 Vgl. ebd. S. 15-16. 
in den Abläufen von Ritualen, die aus den Phasen der Trennung, des Übergangs und der Angliederung bestehen ${ }^{40}$. Das Hauptaugenmerk liegt dabei auf der Phase des Übergangs, der Schwellenphase. Die Eigenschaften der Schwellenphase, die Turner Liminalität nennt, sind für ihn notwendigerweise „unbestimmt ${ }^{\text {“ }}$. Denn Liminalität und Personen, die sich in ihr befinden, schlüpfen durch das „Netz der Klassifikationen, die normalerweise Zustände und Positionen im kulturellen Raum fixieren “42 hindurch. Ihr Status ist gekennzeichnet durch eine temporär limitierte ideelle und kulturelle Ortlosigkeit. ${ }^{43}$ „Schwellenwesen sind weder hier noch da; sie sind weder das eine noch das andere, sondern befinden sich zwischen den vom Gesetz, der Tradition, der Konvention und dem Zeremonial fixierten Positionen. ${ }^{44}$ Die Personen im Zustand der Liminalität besitzen weder Zugehörigkeit zum Status vor der Trennung, aber ebenso wenig gehören sie zum Status nach der gelungenen Angliederung. Sie sind mittendrin, ungestaltet und unbestimmt. ${ }^{45}$ Turner betont aber auch, dass Liminalität ebenso „kulturelle Spielräume für Experimente und Innovationen “ ${ }^{46}$ eröffnen kann. In der Schwellenphase können durch Experimentieren und im Modus des Spiels neue symbolische und kulturelle Formen erprobt, benutzt und wieder verworfen werden. „In liminality [...] new combinations of symbols are tried out, to be discarded or accepted “ ${ }^{47}$

Viele der von Turner festgestellten Kennzeichen der Liminalität lassen sich auf die konkrete Situation moderner Schriftsteller angesichts ihrer Bemühungen, das Unsagbare darzustellen und transzendente Erfahrungen zu beschreiben, adaptieren. Dabei muss aber ein besonderer Umstand beachtet werden. In gewisser Hinsicht sind die Schriftsteller der Moderne in doppelter Weise im Zustand der Liminalität. Zum einen besitzt die transzendente Erfahrung bzw. der konkrete Moment der Konfrontation mit dem Unsagbaren an sich einen liminalen Charakter. Das Übersteigen, das Transzendieren der Alltagserfahrung führt tief in einen liminalen Raum, in dem die fixierten Klassifikationen, Normen und Regeln per se außer Kraft gesetzt sind. Dort bleibt das Individuum angesichts des Unausprechlichen allein mit intensiven Affekten und Emotionen, die, wie bereits beschrieben, unterschiedlicher Couleur sein können. Die Sprache und die Wörter selbst verlieren für die Dauer der konkreten Erfahrung ihre Strukturkraft und die ihnen innewohnende Ordnungsmacht. Zum anderen tritt in der Epoche der Moderne zur liminalen Form der transzendenten Erfahrung noch ein zweites Moment von Liminalität

40 Vgl. Victor Turner, Vom Ritual zum Theater: Der Ernst des menschlichen Spiels, Frankfurt a.M., New York 2009, S. 34. Und ebenso Arnold van Gennep, Übergangsriten (Les rites des passage), Frankfurt a.M., New York 2005, S. 29.

${ }^{41}$ Victor Turner, Das Ritual: Struktur und Anti-Struktur, Frankfurt a.M. 2000, S. 95.

42 Ebd.

43 In traditionellen Stammesgesellschaften wird diese Ortlosigkeit während der Dauer der die Übergangsphase begleitenden Rituale auch räumlich arrangiert.

44 Victor Turner, Das Ritual: Struktur und Anti-Struktur, S. 95.

45 Was Turner im englischen Original-Text mit der zum Schlagwort gewordenen Phrase als between and betwixt bezeichnet. Vgl. hierzu: Victor Turner, The Ritual Process. Structure and Anti-Structure, London 1969, S. 95.

46 Erika Fischer-Lichte, Einleitung zur Aktualität von Turners Studien zum Übergang vom Ritual zum Theater, in: Victor Turner, Vom Ritual zum Theater: Der Ernst des menschlichen Spiels, Frankfurt a.M., New York 2009, S. I-XXIII, hier: S. VII.

47 Victor Turner, Variations on a Theme of Liminality, in: Sally F. Moore, Barbara C. Myerhoff (Hrsg.), Secular Ritual, Assen 1977, S. 36-53, hier: S. 40. 
hinzu. Es ist die Moderne selbst, die viele Züge einer Schwellenphase trägt. Die von Lukács konstatierte metaphysische Obdachlosigkeit oder Edmund Husserls beschriebene Krise der abendländischen Wissenschaft sind Spiegel der manifest werdenden Symptome der Moderne. Sie verweisen auf den großen Umbruchscharakter dieser Epoche, in der religiöse, aber auch gesellschaftliche Werte ihre ehemalige fraglose Gültigkeit verloren haben. Sie sind ebenso Zeugnisse der Tatsache, dass es noch keine allgemeingültigen Antworten gibt auf die drängenden Fragen, die durch die immensen gesellschaftlichen wie wissenschaftlichen Veränderungen in der Moderne gestellt werden. Diese doppelte Form von Liminalität ist die tiefere Ursache der vorher beschriebenen doppelten Sprachlosigkeit, mit der die modernen Schriftsteller konfrontiert sind. Die Liminalität liefert auch eine Erklärung für den paradox anmutenden Rückbezug vieler Autoren auf das fragwürdig gewordene religiöse Vokabular der Tradition. Auf der Schwelle, im Zustand der Liminalität, nutzen die Schriftsteller die sich bietenden Experimentierräume, in denen sie sich losgelöst von allen geltenden Konventionen, angesichts ihrer Erfahrungen mit den Unsagbaren auch mit Sprachmaterial aus dem religiösen Feld ausprobieren können. Dass dabei die alten Konnotationen überschrieben, verändert oder ins Gegenteil verkehrt werden können, gehört zum liminalen Sprachexperiment dazu.

Bezogen auf Liminalität unterscheidet sich aber die Situation der modernen Schriftsteller und Autoren von der der Initianden traditioneller Stammesgesellschaften in einem wichtigen Punkt. Die Schwellenphase in traditionellen Stammesgesellschaften ist eingebunden in ein ganzes Netz konkreter Rituale und strukturiert durch ein komplexes System festgelegter Abläufe und Gebräuche, die den Durchgang der Individuen durch die Liminalitätsperiode begleiten. Schamanen oder Medizinmänner überwachen die ordnungsgemäße, streng ritualisierte Durchführung der verschiedenen Stationen während der Liminalität. In der Moderne des westlichen Abendlandes fehlen dieses Netz und dieses komplexe System, welches behilflich dabei ist, die Übergangsphase zu meistern. Wie bereits erwähnt, ist in der Moderne die traditionelle, religiöse Struktur brüchig und fragwürdig geworden. Bedingt dadurch lassen sich nur wenige erfahrene Begleiter oder Seelenführer finden, die den intensiven Prozess der Konfrontation mit dem Unsagbaren bzw. einer transzendenten Erfahrung, vorrausschauend und unterstützend begleiten können. Die Literaten der Moderne, welche die Mühen auf sich nehmen, ihre Erfahrungen und Erlebnisse zu versprachlichen, sind auf sich allein gestellt. Sie müssen sich allein zurechtfinden. Angesichts einer doppelten Form von Liminalität sind sie gezwungen, sich selbst in eigener Art und Weise den Weg zu bahnen. Diese Wege sind höchst individuell und einzigartig. Sie sind Ausdruck einer je solitären Erfahrung des Unsagbaren.

So kann Blumenbergs Aussage über Kafkas „Brief an den Vater“, dass dieser „eines der wesentlichen Dokumente menschlicher Existenz überhaupt ${ }^{\text {" }}{ }^{48}$ sei, in bestimmter Weise erweitert werden. Die Texte der modernen Schriftsteller, die transzendente Erfahrungen beschreiben und darstellen, sind zugleich wichtige Dokumente der menschlichen Existenz. Sie geben Zeugnis von der immerwährenden Möglichkeit des Menschen, eine die Alltagswirklichkeit übersteigende Erfahrung zu machen, die den Menschen an einen Bereich führt, der sich dem Sagbaren und der Sprache entzieht. Auch geben sie Zeugnis von der Anstrengung und dem Kampf des Menschen in seiner jeweils konkreten geschichtlichen Situation, diese Erfahrung

48 Hans Blumenberg, Der absolute Vater, S. 282. 
trotz aller Unmöglichkeit doch in Wort zu fassen. Sie spiegeln den Willen des Dichters und Schriftstellers wider, für seine Mitmenschen das Unsagbare doch zu sagen und dem Namenlosen Namen, Worte und auch Bedeutung zu verleihen.

\section{Literatur}

Augustinus, Bekenntnisse, Berlin, Darmstadt 1968.

Blumenberg, Hans, Der absolute Vater, in: „Hochland“ 45 (1952/53), S. 282-284.

Blumenberg, Hans, Paradigmen zu einer Metaphorologie. Kommentar von Anselm Haverkamp, Frankfurt a.M. 1998.

Colpe, Carsten, Über das Heilige. Versuch, seine Verkennung kritisch vorzubeugen, Frankfurt a.M. 1990.

Fischer-Lichte, Erika, Einleitung zur Aktualität von Turners Studien zum Übergang vom Ritual zum Theater, in: Victor Turner, Vom Ritual zum Theater: Der Ernst des menschlichen Spiels, Frankfurt a.M., New York 2009, S. I-XXIII.

van Gennep, Arnold, Übergangsriten (Les rites des passage), Frankfurt a.M., New York 2005.

Goltschnigg, Dietmar, Mystische Tradition im Roman Robert Musils. Martin Bubers „Ekstatische Konfessionen“ im „Mann ohne Eigenschaften“, Heidelberg 1974.

Hölderlin, Friedrich, Wie wenn am Feiertage, in: ders., Sämtliche Gedichte, Herausgegeben von Detlev Lüders, Wiesbaden1989, S. 300-302.

Klein, Johannes, Kampf um Gott in der deutschen Dichtung, Witten, Berlin 1974.

Lukács, Georg, Die Theorie des Romans. Ein geschichtsphilosophischer Versuch über die Formen der großen Epik, Darmstadt, Neuwied 1982.

Meister, Eckhart, Werke I. Texte und Übersetzungen von Josef Quint. Herausgegeben und kommentiert von Niklas Largier, Frankfurt a.M. 2006.

Meyer, Theo, Nietzsche und die Kunst, Tübingen 1993.

Musil, Robert, Der Mann ohne Eigenschaften. Bd. 2. Aus dem Nachlass. Herausgegeben von Adolf Frisé, Reinbek bei Hamburg 2009.

Nietzsche, Friedrich, Vorwort an Richard Wagner. Die Geburt der Tragödie. Oder: Griechenthum und Pessimismus, in: ders., Die Geburt der Tragödie. Unzeitgemäße Betrachtungen I-III (1872-1874). Nietzsches Werke. Kritische Gesamtausgabe. Giorgio Colli und Mazzino Montinari. Abt. 3, Bd. 1, Berlin 1972, S. 5-150.

Nietzsche, Friedrich, Die Fröhliche Wissenschaft, in: ders., Morgenröte. Idyllen aus Messina. Fröhliche Wissenschaft. Kritische Studienausgabe, Bd. 3. Herausgegeben von Giorgio Colli und Mazzino Montinari, Berlin, New York, München 1988, S. 343-653.

Otto, Rudolf, Das Heilige. Über das Irrationale in der Idee des Göttlichen und sein Verhältnis zum Rationalen, München 2004.

Rilke, Rainer Maria, Neue Gedichte, Leipzig 1907.

Rilke, Rainer Maria, Duineser Elegien, Leipzig 1923.

Rimbaud, Arthur, Sämtliche Dichtungen. Zweisprachige Ausgabe, 2004 München.

Stieg, Gerald, Sakralisierung durch Dichtung bei Rainer Maria Rilke und Georg Trakl, in: Albert Meier, Alessandro Cosstazza, Gérard

Laudin (Hrsg.), Kunstreligion. Ein ästhetisches Konzept der Moderne in seiner Entfaltung. Bd. 2: Die Radikalisierung des Konzepts nach 1850, Berlin, Boston 2012, S. 265-279.

Sudbrack, Josef, Mystik. Sinnsuche und die Erfahrung des Absoluten, Darmstadt 2002.

Trakl, Georg, Gedichte, Leipzig 1913. 
Turner, Victor, The Ritual Process. Structure and Anti-Structure, London 1969.

Turner, Victor, Variations on a Theme of Liminality, in: Sally F. Moore, Barbara C. Myerhoff(Hrsg.), Secular Ritual, Assen 1977, S. 36-53.

Turner, Victor, Das Ritual. Struktur und Anti-Struktur, Frankfurt a.M. 2000.

Turner, Victor, Vom Ritual zum Theater. Der Ernst des menschlichen Spiels, Frankfurt a.M., New York 2009. 